\title{
Immune determinants of COVID-19 disease presentation and severity
}

\author{
Petter Brodin (101,2凶
}

COVID-19, caused by SARS-CoV-2 infection, is mild to moderate in the majority of previously healthy individuals, but can cause life-threatening disease or persistent debilitating symptoms in some cases. The most important determinant of disease severity is age, with individuals over 65 years having the greatest risk of requiring intensive care, and men are more susceptible than women. In contrast to other respiratory viral infections, young children seem to be less severely affected. It is now clear that mild to severe acute infection is not the only outcome of COVID-19, and long-lasting symptoms are also possible. In contrast to severe acute COVID-19, such 'long COVID' is seemingly more likely in women than in men. Also, postinfectious hyperinflammatory disease has been described as an additional outcome after SARS-CoV-2 infection. Here I discuss our current understanding of the immunological determinants of COVID-19 disease presentation and severity and relate this to known immune-system differences between young and old people and between men and women, and other factors associated with different disease presentations and severity.

T he SARS-CoV-2 virus infects humans via droplets, and to some extent, aerosols. In symptomatic adults, the disease typically presents after $2-14$ days of incubation as a respiratory illness with fever, cough, headache, myalgia and in some cases intestinal symptoms ${ }^{2}$. A growing number of studies are pointing toward asymptomatic infection in a significant fraction of individu$\mathrm{als}^{3}$, and as many as half of all transmission events occur from presymptomatic and asymptomatic individuals ${ }^{4}$.

In this Perspective, I discuss what we know about the immune response to SARS-CoV-2 infection and how this might explain different disease presentations and disease severity by considering known immunological differences between the groups that are most commonly affected.

\section{COVID-19 disease courses}

Mild and severe acute COVID-19. It is clear that the outcome of infection with the severe acute respiratory syndrome coronavirus 2 (SARS-CoV-2) varies broadly, with the majority of young individuals experiencing mild disease ${ }^{5}$. Also, sex is an important; men are over-represented among patients with severe disease, presumably due to differences in the elicited immune responses ${ }^{6}$. Comorbidities such as obesity, hypertensive disease, chronic obstructive pulmonary disease and cardiovascular disease are all associated with severe COVID-19 disease ${ }^{2}$. Higher SARS-CoV-2 copy numbers at diagnosis have been reported in patients with severe COVID-19 than in those with mild COVID-19 (ref. ${ }^{7}$ ). Smoking is yet another risk factor: cigarette smoke induces expression of angiotensin-converting enzyme 2 (ACE2), which allows SARS-CoV-2 to enter cells, and could possibly influence viral invasion ${ }^{8}$ beyond its negative effects on overall lung function.

Despite the increased risk of severe disease with increased age, a minor subset of young and middle-aged individuals present with severe COVID-19 disease characterized by poor oxygen saturation and massive inflammatory responses in the lung. Such cases need urgent management and intensive care, and several studies have attempted to unravel the mediators of such hyperinflammatory disease presentation ${ }^{9-13}$.
Long COVID. Apart from the differences in severity among patients with acute COVID-19, it is now clear that a number of other outcomes are possible after an initial infection with SARS-CoV-2. After a long period of intensive care and mechanical ventilation, general anesthesia and severe illness, it is not surprising that long rehabilitation periods are needed ${ }^{14}$. However, it is now also clear that some individuals with milder initial symptoms of COVID-19 can suffer from variable and debilitating symptoms for many months after the initial infection ${ }^{15,16}$. This condition is popularly referred to as long COVID. An exact definition is lacking, but typically symptoms with a duration $>2$ months are considered long COVID. The condition involves a range of symptoms such as persistent fatigue, myalgia, autonomic dysregulation manifested as postural orthostatic tachycardia syndrome, abnormal thermoregulation, intestinal disturbances and skin manifestations $s^{17}$. This post-COVID syndrome bears resemblance to postinfectious syndromes that followed outbreaks of chikungunya ${ }^{18}$ and Ebola ${ }^{19}$, for example, and selected symptoms overlap with myalgic encephalomyelitis, a disease that is also often triggered by infection and immune activation ${ }^{20}$ and manifests as a dysregulated autonomic nervous system and perturbed immune parameters ${ }^{21}$. More research is needed to understand the pathogenesis of all of these postinfectious conditions, and long COVID offers a unique opportunity to perform such studies in larger numbers of individuals, all infected by the same virus during a limited time frame.

Multisystem inflammatory syndrome associated with COVID19. Another rare and serious postinfectious condition that can occur 2-6 weeks after SARS-CoV-2 infection is the multisystem inflammatory syndrome associated with COVID-19, first described in children (MIS-C) $)^{22-24}$, and more recently in young adults (MIS-A) ${ }^{25}$. This hyperinflammatory syndrome shares clinical features with Kawasaki disease ${ }^{26}$, but affects children who are older than the typical patient with Kawasaki disease and who more often present with intestinal involvement and myocardial failure and shock. There is also significant clinical overlap in presentation with toxic shock syndrome $^{22}$ or septic shock. Subgroups of children affected by MIS-C

'Science for Life Laboratory, Department. of Women's and Children's Health, Karolinska Institutet, Solna, Sweden. ${ }^{2}$ Pediatric Rheumatology, Karolinska University Hospital, Solna, Sweden. $\varpi_{e}$-mail: petter.brodin@ki.se 
are being described ${ }^{27}$, and optimal management is being worked out by collaborative networks of pediatricians. Most MIS-C patients are treated with strong immunomodulatory regimens such as high-dose steroids, intravenous immunoglobulins and anti-cytokine therapies coupled with anti-coagulation to counter the microangiopathy and activation of both complement and coagulation cascades during the hyperinflammatory disease phase ${ }^{28,29}$. The pathogenesis of MIS-C is unknown, but a delay of 2-6 weeks from initial SARS-CoV-2 infection indicates a role for adaptive immune responses and specific autoantibodies have been proposed ${ }^{30,31}$.

\section{Viral recognition and innate immune responses}

Viral entry. SARs-CoV-2 infects cells by attaching to the principal viral entry receptor, ACE2 (ref. ${ }^{32}$ ). The expression of this receptor has been reported in single-cell messenger-RNA-sequencing data on epithelial cells in the oral mucosa ${ }^{33}$, liver, kidney, intestine and heart $^{34}$, and at the protein level in alveolar epithelial cells ${ }^{35}$, although the tissue distribution of protein expression differs to some extent ${ }^{36}$. Several reports have shown abundant expression of ACE2 in the intestinal epithelium leading to viral shedding via feces ${ }^{37}$, while ACE2 does not seem to be expressed by cells of the immune system ${ }^{38}$.

Innate immune responses. SARS-CoV-2, like the related SARS-CoV and Middle East respiratory syndrome coronavirus (MERS-CoV), is a single-stranded RNA virus. After entering a target cell, the virus is recognized by pattern recognition receptors such as Toll-like receptors 3, 7, 8 and 9 and viral-infection sensors RIG-I and MDA5 (ref. ${ }^{39}$ ), and viral recognition induces the type I interferon (IFN) response program and IFN-stimulated genes ${ }^{40}$ (Fig. 1a). The TLR3 response triggers transcription of the NLR family pyrin domain containing 3 (NLRP3) gene, which together with other cellular responses to viral infection-such as the formation of reactive oxidative species, calcium flux from cytoplasmic storages, protein aggregation and the release of danger-associated patterns-contributes to the activation of the NLRP3 inflamasome ${ }^{41}$ and likely other inflammasome complexes. The NLRP3 inflammasome induces caspase-1-dependent cleavage and release of key proinflammatory cytokines interleukin-1 $\beta$ (IL-1 $\beta$ ) and IL-18, and triggers gasdermin D-mediated pyroptotic cell death. The extent of NLRP3 activation correlates with COVID-19 disease severity ${ }^{42}$ (Fig. 1b). As a result of pyroptotic cell death, the enzyme lactate dehydrogenase (LDH) is released. Elevated LDH levels have been observed in the blood of patients with COVID-19, and levels of this enzyme correlate with disease severity ${ }^{2}$. Together, these data suggest that inflammasome activation is an important feature of COVID-19 (ref. ${ }^{43}$ ) (Fig. 1b). This pathway also triggers the coagulation cascade, for example via the extracellular release of gasdermin $\mathrm{D}^{44}$, and coagulopathy and severe thrombotic events are common in patients with severe COVID-19 (ref. ${ }^{45}$ ). A similar activation of the coagulation cascade and elevated LDH levels are also seen in patients with MIS-C ${ }^{22}$, but not in patients with long $\operatorname{COVID}^{46}$, indicating differences in the underlying pathogenesis.

A characteristic feature of SARS-CoV and MERS-CoV viruses is their ability to inhibit and delay the induction of type I IFN by infected cells, which contributes to the immunopathology associated with such infections ${ }^{47,48}$. Also, SARS-CoV-2 is able to inhibit the type I IFN responses in infected cells, leading to delayed or overall suppressed type I IFN responses ${ }^{49,50}$. This allows the virus to replicate and induce more tissue damage, and triggers a more exuberant immune response as the immune system struggles to limit viral replication and to manage dying and dead cells. Immune pathology continues as inflammatory cells flow into the lung and produce large amounts of proinflammatory cytokines, further escalating the situation (Fig. 1c). Such imbalanced immune responses, caused in part by the impaired early type I IFN responses, are the most likely determinant of the overall severity of acute COVID-19 (refs. ${ }^{50-53}$ ).
This is further emphasized by recent results from the COVID Human Genetic Effort ${ }^{54}$ (https://www.covidhge.com/), which found that inborn errors in the type I IFN pathway ${ }^{55}$, or the presence of neutralizing autoantibodies to type I IFNs ${ }^{56}$, were strongly over-represented among individuals who developed life-threatening COVID-19. Whether imbalanced or impaired innate responses also contribute to the development of other disease manifestations such as MIS-C and long COVID remains to be determined.

\section{Adaptive immune responses}

Serological tests for SARS-CoV-2 have been the subject of much discussion and conflicting results during the course of this pandemic so far. However, with time it has become apparent that the adaptive immune responses induced by SARS-CoV-2 infection largely follow the expected patterns based on what is known from other comparable viral infections, with $>90 \%$ of infected individuals seroconverting a few weeks after initial infection ${ }^{57,58}$. Presence of anti-spike IgG antibodies were associated with protection from reinfection in a UK cohort of health-care workers at high risk of exposure ${ }^{59}$.

$\mathrm{T}$ cell responses to the SARS-CoV-2 spike protein correlate with $B$ cell responses to the same protein and are detectable in nearly all convalescent patients with COVID-19 (ref. ${ }^{60}$ ). T cell reactivity to SARS-CoV-2 can also be detected in unexposed individuals, presumably due to cross-reactive immunity to common-cold coronaviruses $^{60}$ or to other antigens, as has been shown for other virus-specific $\mathrm{T}$ cells ${ }^{61}$. Another study has reported SARS-CoV2-reactive $\mathrm{T}$ cells in patients who survived the SARS epidemic in 2003, but also in unexposed individuals; interestingly, such responses preferentially targeted epitopes different from the ones in convalescent patients with COVID-19, and were not homologous with common-cold coronaviruses, but conserved among animal coronaviruses $^{62}$.

Antibody-dependent enhancement (ADE), a phenomenon that has been described for infections with viruses such as dengue ${ }^{63}$, has been proposed as a possible mechanism of severe COVID-19. $\mathrm{ADE}$ occurs when antibodies target a virus without neutralizing it, for example if the antibody is raised against a different serotype of the virus or when the antibody fails to block viral entry. Then, the antibody might facilitate Fc-receptor-mediated endocytosis of the virus and enhanced viral replication, and massive inflammatory responses. This has been described to occur for MERS ${ }^{64}$, but no clear evidence of ADE as a cause of severe SARS-CoV-2 infection has been communicated. Reinfections have been reported, and in a few instances, the second infection was more severe than the first, but serological responses suggest that patients never seroconverted after initial infection and ADE is a less likely cause of a more severe second infection ${ }^{65}$.

The role of pre-existing immunity to common-cold coronaviruses is another possible determinant of COVID-19 disease severity $^{66}$. T cell reactivity is found in unexposed individuals and has been linked to prior exposures to common-cold coronaviruses ${ }^{67}$. Also, IgG that is specific to SARS-CoV-2 spike protein has been found in unexposed individuals, particularly in children and young adults, and some of these had neutralizing activity against SARS-CoV-2, indicating a potentially protective effect against severe COVID-19 (ref. ${ }^{68}$ ). Another study also identified such antibodies but found no evidence for a protective effect against COVID-19 (ref. ${ }^{69}$ ). Cross-reactive antibodies are also more frequently found in serum samples collected in sub-Saharan Africa prior to the COVID-19 pandemic ${ }^{70}$, indicating a possible explanation for the surprisingly low number of severe COVID-19 cases seen on this continent. Whether there is a role for cross-reactive antibodies or T cells, or the absence of such features, in determining other disease manifestations, such as MIS-C or long COVID, remains to be seen. Children who develop MIS-C have detectable IgG responses without obvious differences from convalescent children without MIS-C ${ }^{29,71,72}$, 


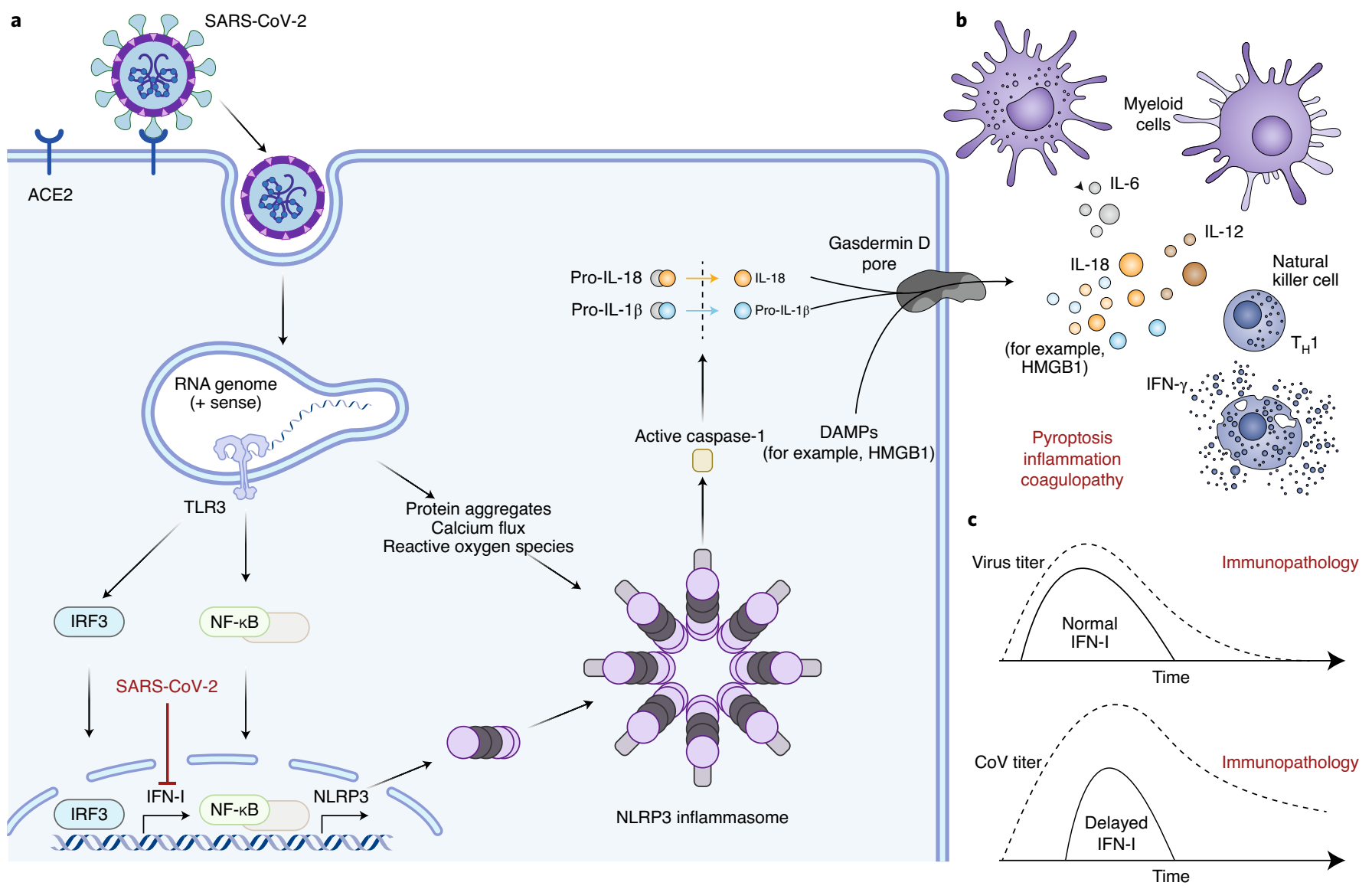

Fig. 1 | Coronavirus recognition and immune response. a, SARS-CoV-2 viruses bind to the ACE2 receptor for cell entry. Viral RNA is recognized by TLR3, which triggers transcriptional responses and cytoplasmic changes that activate the NLRP3 inflammasome. This leads to cleavage of precursor IL-1 $\beta$ (pro-IL-1 $\beta$ ), pro-IL-18 and gasdermin D, allowing secretion of IL-1 $\beta$ and IL-18. These changes collectively induce pyroptosis, inflammation and coagulopathy b, Secreted IL-18 together with IL-12 from myeloid cells stimulate $T_{H} 1$ immunity and natural killer cells to secrete IFN- $\gamma$. c, A key feature of coronaviruses (MERS-CoV, SARS-CoV) is a capability to inhibit and delay the type I IFN response, leading to increased viral replication and severe immunopathology.

although one study indicated subtle IgG-subclass and functional differences between children with MIS-C and those without MIS- $\mathrm{C}^{73}$.

\section{Known immunological differences between high- and low-risk individuals}

The risk of developing severe COVID-19 increases steeply above age 70 , and also with the severity of obesity and other risk factors. Men have a much greater risk of severe acute COVID-19 than women, whereas women are over-represented among patients who develop long COVID $^{15}$. The infection differs from many other respiratory infections in that children are seemingly able to cope, even in the very first years of life, without developing severe respiratory disease except in a few rare cases. The known immunological differences between young and old people and between men and women should help us further unravel the immunological mechanisms behind disease presentation and severity.

Sex differences. As mentioned above, type I IFN responses are critical determinants of disease severity during acute SARS-CoV-2 infection, and the virus has developed methods for subverting these responses. Women elicit stronger type I IFN responses upon stimulation with TLR7 ligand ${ }^{74}$ and develop stronger vaccine responses ${ }^{75}$, but also more side effects, and have better survival rates for a number of acute infections than do men ${ }^{76}$. These sex differences are seen even in boys and girls before puberty-pointing towards genetic, rather than hormonal, differences. It is worth noting that a common sensor of viruses, TLR7, is expressed on the X chromosome, providing a possible difference in gene-dosage effect between men and women ${ }^{77}$. Interestingly, the neutralizing autoantibodies to type I IFN found in patients with severe COVID-19 were much more abundant in men than in women, but the reasons for this are elusive $^{56}$. Immune-response differences have also been reported between male and female patients with COVID-19 (ref. ${ }^{6}$ ), and collectively these sex differences could explain the overall susceptibility of male patients to developing severe acute COVID-19. MIS-C is quite evenly distributed between boys and girls ${ }^{22}$, whereas long COVID is more prevalent in female patients ${ }^{15,16}$. It is also important to consider whether social factors and differing exposure play a part in sex differences.

Age differences. If type I IFN responses were the sole determinants of COVID-19 severity, one would expect young children to be highly susceptible because both newborn and young children produce lower amounts of type I IFN upon stimulation through various viral-sensing pathways ${ }^{78}$. The low risk of severe SARS-CoV-2 disease in young children also differs from that of other respiratory viral infections like the flu ${ }^{79}$, and points toward other protective mechanisms in young children. The immune systems of young children are accustomed to facing novel challenges, whereas older individuals rely more on memory responses. The thymus decreases its output of naive $\mathrm{T}$ cells and involutes at a rate of about $3 \%$ per year, and some data indicate more rapid involution in boys than in 


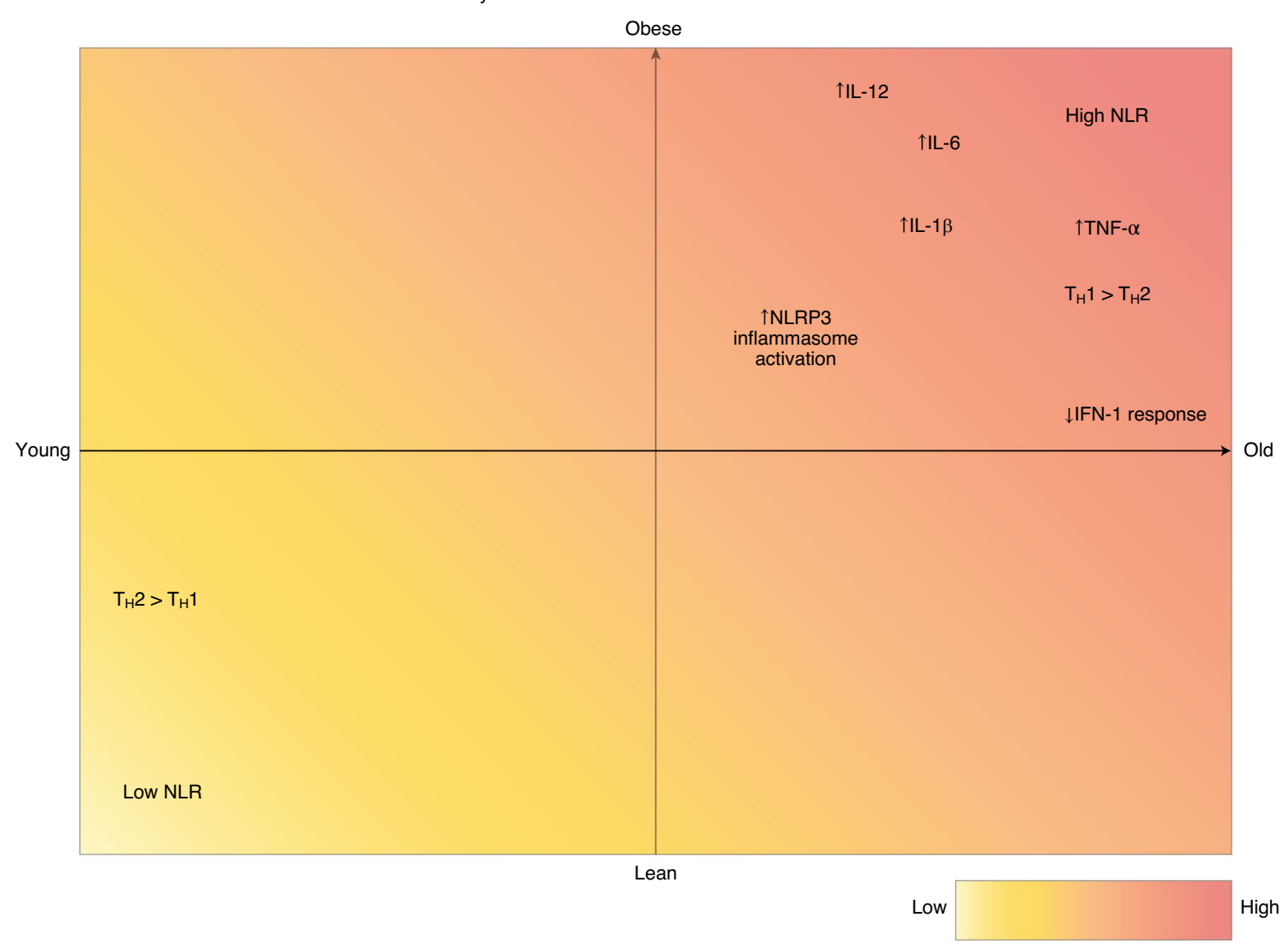

Fig. 2 | Immune-system parameters associated with obesity, aging and severe COVID-19. The gradient illustrates the risk of developing severe COVID-19 in relation to known immune system changes with age ( $x$ axis) and obesity ( $y$ axis).

girls ${ }^{80}$. Cross-reactive antibodies to common-cold coronaviruses are one possible explanation; another possibility is that constitutive differences in immune system states between young and old people are of importance. One example could be the skewing of $\mathrm{T}$ cells from $\mathrm{T}$ helper type $1\left(\mathrm{~T}_{\mathrm{H}} 1\right)$ toward more $\mathrm{T}_{\mathrm{H}} 2$ in young children ${ }^{81}$ (Fig. 2$)$.

Disease severity in COVID-19 also correlates with neutrophilto-lymphocyte ratio (NLR) ${ }^{82}$, a metric reflecting immune-cell composition that is frequently studied across populations and disease conditions as a surrogate marker of systemic inflammation. The NLR ratio positively correlates with advancing age $e^{83}$ and with the degree of obesity, especially in the context of metabolic syndromes and type 2 diabetes $^{84}$. As such, the NLR ratio is indicative of low-grade inflammation, 'inflammaging' and obesity-associated inflammation, and is a poor prognostic factor in COVID-19 (Fig. 2). This observation indicates that individuals with such underlying immune-system conditions either fail to develop productive antiviral immune responses or are more prone to develop uncontrolled, exuberant responses upon infection, leading to hyperinflammation and acute respiratory distress syndrome, characteristic of severe COVID-19 (Fig. 2). Older individuals typically produce weaker type I IFN responses upon viral infection, which further worsen the situation ${ }^{85}$. Also, additional markers of inflammaging and obesity-associated inflammation have been shown to be predictive of a severe COVID-19 course, such as NLRP3 activation ${ }^{43}$, IL-6, IL- 12 and IL- $1 \beta$ secretion $^{86}$ and danger-associated molecular patterns, including high mobility group box 1 (HMGB1) ${ }^{87}$ (Fig. 2).

Immunodeficiencies. Since the beginning of the pandemic, there have been grave concerns over the risk of developing severe COVID-19 for individuals with immunodeficiencies or those tak- ing immunosuppressive therapies. One systematic review found no statistically significant increased risk of severe COVID-19 in immunosuppressed patients ${ }^{88}$, but other studies have shown an increased risk for patients with solid-organ transplants and some patients with cancer ${ }^{89}$. Patients with cancer treated with checkpoint inhibitors are at particularly high risk of severe COVID-19, according to another recent report ${ }^{90}$. It is important to note that type and degree of immunosuppression likely varies substantially among heterogeneous patient groups, and more detailed subset analyses are needed. This is also highlighted by an Italian study of patients with different forms of primary antibody deficiencies, in which patients with combined variable immunodeficiency, often associated with low-grade inflammation, developed severe COVID-19, while patients with similarly low antibody levels due to other forms of inborn errors of immunity generally experienced milder course of disease ${ }^{91}$.

Future directions. In summary, COVID-19 can develop into a life-threatening hyperinflammatory disease in rare cases, and global efforts are ongoing to better understand productive immune responses against the SARS-CoV-2 virus and the immunopathological mechanisms underlying severe disease. At the same time, additional disease outcomes, such as MIS-C, MIS-A and long COVID, are becoming increasingly known. By considering known differences between high- and low-risk individuals for each of these conditions, we will be able to design optimal studies contrasting such patient groups and their immune responses. The origin of the different disease trajectories upon SARS-CoV-2 infection can likely be traced back to the early stages of infection, as illustrated by the essential role for type I IFN responses during acute COVID. With this Perspective, I hope to open up a discussion on the different 
disease manifestations, their shared and distinguishing features and affected populations and immunological features.

Received: 1 May 2020; Accepted: 3 December 2020; Published online: 13 January 2021

\section{References}

1. van Doremalen, N. et al. Aerosol and surface stability of SARS-CoV-2 as compared with SARS-CoV-1. N. Engl. J. Med. 382, 1564-1567 (2020).

2. Huang, C. et al. Clinical features of patients infected with 2019 novel coronavirus in Wuhan, China. Lancet 395, 497-506 (2020).

3. Wölfel, R. et al. Virological assessment of hospitalized patients with COVID-2019. Nature 581, 465-469 (2020)

4. Moghadas, S. M. et al. The implications of silent transmission for the control of COVID-19 outbreaks. Proc. Natl Acad. Sci. USA 117, 17513-17515 (2020).

5. Brodin, P. Why is COVID-19 so mild in children? Acta Paediatr. 109, 1082-1083 (2020)

6. Takahashi, T. et al. Sex differences in immune responses that underlie COVID-19 disease outcomes. Nature 588, 315-320 (2020).

7. Liu, Y. et al. Viral dynamics in mild and severe cases of COVID-19. Lancet Infect. Dis. 20, 656-657 (2020)

8. Smith, J. C. et al. Cigarette smoke exposure and inflammatory signaling increase the expression of the SARS-CoV-2 receptor ACE2 in the respiratory tract. Dev. Cell 53, 514-529(2020).

9. Mehta, P. et al. COVID-19: consider cytokine storm syndromes and immunosuppression. Lancet 395, 1033-1034 (2020).

10. Cao, X. COVID-19: immunopathology and its implications for therapy. Nat. Rev. Immunol. 20, 269-270 (2020).

11. Mathew, D. et al. Deep immune profiling of COVID-19 patients reveals distinct immunotypes with implications for therapeutic interventions. Science 369, eabc8511 (2020).

12. Lucas, C. et al. Longitudinal immunological analyses reveal inflammatory misfiring in severe COVID-19 patients. Nature 584, 463-469 (2020).

13. Rodriguez, L. et al. Systems-level immunomonitoring from acute to recovery phase of severe COVID-19. Cell Rep. Med. 1, 100078 (2020).

14. Carfi, A. et al. Persistent symptoms in patients after acute COVID-19. JAMA 324, 603-605 (2020)

15. Dennis, A. et al. Multi-organ impairment in low-risk individuals with long COVID. Preprint at medRxiv https://doi.org/10.1101/2020.10.14.20212555 (2020).

16. Ludvigsson, J. F. Case report and systematic review suggest that children may experience similar long-term effects to adults after clinical COVID-19. Acta Paediatr. https://doi.org/10.1111/apa.15673 (2020).

17. Davido, B., Seang, S., Tubiana, R. \& de Truchis, P. Post-COVID-19 chronic symptoms: a post-infectious entity? Clin. Microbiol. Infec. 26, 1448-1449 (2020).

18. Guillot, X., Ribera, A. \& Gasque, P. Chikungunya-induced arthritis in Reunion Island: a long-term observational follow-up study showing frequently persistent joint symptoms, some cases of persistent chikungunya immunoglobulin $\mathrm{M}$ positivity, and no anticyclic citrullinated peptide seroconversion after 13 years. J. Infect. Dis. 222, 1740-1744 (2020).

19. Clark, D. V. et al. Long-term sequelae after Ebola virus disease in Bundibugyo, Uganda: a retrospective cohort study. Lancet Infect. Dis. 15, 905-912 (2015).

20. Hickie, I. et al. Post-infective and chronic fatigue syndromes precipitated by viral and non-viral pathogens: prospective cohort study. BMJ 333, 575 (2006)

21. Rodriguez, L. S. T. et al. Achieving symptom relief in patients with Myalgic encephalomyelitis by targeting the neuro-immune interface and inducing disease tolerance. Preprint at bioRxiv https://doi. org/10.1101/2020.02.20.958249 (2020).

22. Whittaker, E. et al. Clinical characteristics of 58 children with a pediatric inflammatory multisystem syndrome temporally associated with SARS-CoV-2. JAMA 324, 259-269 (2020).

23. Belot, A. et al. SARS-CoV-2-related paediatric inflammatory multisystem syndrome, an epidemiological study, France, 1 March to 17 May 2020. Euro. Surveill. 25, 2001010 (2020).

24. Toubiana, J. et al. Kawasaki-like multisystem inflammatory syndrome in children during the COVID-19 pandemic in Paris, France: prospective observational study. BMJ 369, m2094 (2020).

25. Morris, S. B. et al. Case series of multisystem inflammatory syndrome in adults associated with SARS-CoV-2 infection-United Kingdom and United States, March-August 2020. Morbidity Mortal. Wkly Rep. 69, 1450-1456 (2020).

26. Marrani, E., Burns, J. C. \& Cimaz, R. How should we classify Kawasaki disease? Front Immunol. 9, 2974 (2018).

27. Corwin, D. J. et al. Distinguishing multisystem inflammatory syndrome in children from Kawasaki disease and benign inflammatory illnesses in the SARS-CoV-2 pandemic. Pediatr. Emerg. Care 36, 554-558 (2020).
28. Diorio, C. et al. Multisystem inflammatory syndrome in children and COVID-19 are distinct presentations of SARS-CoV-2. J. Clin. Invest. 130, 5967-5975 (2020).

29. Consiglio, C. R. et al. The immunology of multisystem inflammatory syndrome in children with COVID-19. Cell 183, 968-981 (2020).

30. Consiglio, C. R. \& Brodin, P. Stressful beginnings with long-term consequences. Cell 180, 820-821 (2020).

31. Gruber, C. et al. Mapping systemic inflammation and antibody responses in multisystem inflammatory syndrome in children (MIS-C). Cell 183, 982-995 (2020).

32. Hoffmann, M. et al. SARS-CoV-2 cell entry depends on ACE2 and TMPRSS2 and is blocked by a clinically proven protease inhibitor. Cell 181, 271-280 (2020)

33. Xu, H. et al. High expression of ACE2 receptor of 2019-nCoV on the epithelial cells of oral mucosa. Int. J. Oral. Sci. 12, 8 (2020)

34. Qi, F., Qian, S., Zhang, S. \& Zhang, Z. Single cell RNA sequencing of 13 human tissues identify cell types and receptors of human coronaviruses. Biochem. Bioph. Res. Co. 526, 135-140 (2020).

35. Hamming, I. et al. Tissue distribution of ACE2 protein, the functional receptor for SARS coronavirus. A first step in understanding SARS pathogenesis. J. Pathol. 203, 631-637 (2004).

36. Hikmet, F. et al. The protein expression profile of ACE2 in human tissues Mol. Syst. Biol. 16, e9610 (2020).

37. Xu, Y. et al. Characteristics of pediatric SARS-CoV-2 infection and potential evidence for persistent fecal viral shedding. Nat. Med. 26, 502-505 (2020).

38. Uhlen, M. et al. A genome-wide transcriptomic analysis of protein-coding genes in human blood cells. Science 366, eaax9198 (2019).

39. Lim, Y., Ng, Y., Tam, J. \& Liu, D. Human coronaviruses: a review of virus-host interactions. Diseases 4, 26 (2016).

40. Fitzgerald, K. A. \& Kagan, J. C. Toll-like receptors and the control of immunity. Cell 180, 1044-1066 (2020).

41. Nieto-Torres, J. L. et al. Severe acute respiratory syndrome coronavirus E protein transports calcium ions and activates the NLRP3 inflammasome. Virology 485, 330-339 (2015).

42. Rodrigues, T.S. et al. Inflammasomes are activated in response to SARS-CoV-2 infection and are associated with COVID-19 severity in patients. J. Exp. Med. 218, e20201707 (2021)

43. Han, Y. et al. Lactate dehydrogenase, a risk factor of severe COVID-19 patients. Aging 12, 11245-11258 (2020).

44. $\mathrm{Wu}, \mathrm{C}$. et al. Inflammasome activation triggers blood clotting and host death through pyroptosis. Immunity 50, 1401-1411 (2019).

45. Middeldorp, S. et al. Incidence of venous thromboembolism in hospitalized patients with COVID-19. J. Thromb. Haemost. 18, 1995-2002 (2020).

46. Greenhalgh, T., Knight, M., A'Court, C., Buxton, M. \& Husain, L. Management of post-acute COVID-19 in primary care. BMJ 370, m3026 (2020).

47. Züst, R. et al. Ribose 2'-O-methylation provides a molecular signature for the distinction of self and non-self mRNA dependent on the RNA sensor Mda5. Nat. Immunol. 12, 137-143 (2011).

48. Spiegel, M. et al. Inhibition of beta interferon induction by severe acute respiratory syndrome coronavirus suggests a two-step model for activation of interferon regulatory factor 3. J. Virol. 79, 2079-2086 (2005).

49. Miorin, L. et al. SARS-CoV-2 Orf6 hijacks Nup98 to block STAT nuclear import and antagonize interferon signaling. Proc. Natl. Acad Sci. USA 117, 28344-28354 (2020).

50. Arunachalam, P. S. et al. Systems biological assessment of immunity to mild versus severe COVID-19 infection in humans. Science 369, 1210-1220 (2020)

51. Blanco-Melo, D. et al. Imbalanced host response to SARS-CoV-2 drives development of COVID-19. Cell 181, 1036-1045 (2020).

52. Lucas, C. et al. Longitudinal analyses reveal immunological misfiring in severe COVID-19. Nature 584, 463-469 (2020).

53. Vabret, N. et al. Immunology of COVID-19: current state of the science. Immunity 52, 910-941 (2020).

54. Casanova, J.-L., Su, H. C. \& COVID Human Genetic Effort. A global effort to define the human genetics of protective immunity to SARS-CoV-2 infection. Cell 181, 1194-199 (2020).

55. Zhang, Q. et al. Inborn errors of type I IFN immunity in patients with life-threatening COVID-19. Science 370, eabd4570 (2020).

56. Bastard, P. et al. Auto-antibodies against type I IFNs in patients with life-threatening COVID-19. Science 370, eabd4585 (2020).

57. Gudbjartsson, D. F. et al. Humoral immune response to SARS-CoV-2 in Iceland. N. Engl. J. Med. 383, 1724-1734 (2020).

58. Wajnberg, A. et al. Robust neutralizing antibodies to SARS-CoV-2 infection persist for months. Science 370, eabd7728 (2020).

59. Lumley, S. F. et al. Antibodies to SARS-CoV-2 are associated with protection against reinfection. Preprint at medRxiv https://doi. org/10.1101/2020.11.18.20234369 (2020).

60. Grifoni, A. et al. Targets of T cell responses to SARS-CoV-2 coronavirus in humans with COVID-19 disease and unexposed individuals. Cell 181, 1489-1501 (2020) 
61. Su, L. F., Kidd, B. A., Han, A., Kotzin, J. J. \& Davis, M. M. Virus-specific CD4+ memory-phenotype $\mathrm{T}$ cells are abundant in unexposed adults. Immunity 38, 373-83 (2013).

62. Bert, N. L. et al. SARS-CoV-2-specific T cell immunity in cases of COVID-19 and SARS, and uninfected controls. Nature 584, 457-462 (2020).

63. Tetro, J. A. Is COVID-19 receiving ADE from other coronaviruses? Microbes Infect. 22, 72-73 (2020).

64. Wan, Y. et al. Molecular mechanism for antibody-dependent enhancement of coronavirus entry. J. Virol. 94, e02015-e02019 (2019).

65. Iwasaki, A. What reinfections mean for COVID-19. Lancet Infect. Dis. https:// doi.org/10.1016/S1473-3099(20)30783-0 (2020).

66. Lipsitch, M., Grad, Y. H., Sette, A. \& Crotty, S. Cross-reactive memory T cells and herd immunity to SARS-CoV-2. Nat. Rev. Immunol. 20, 709-713 (2020).

67. Mateus, J. et al. Selective and cross-reactive SARS-CoV-2 T cell epitopes in unexposed humans. Science 370, 89-94 (2020).

68. Ng, K. W. et al. Preexisting and de novo humoral immunity to SARS-CoV-2 in humans. Science 370, eabe1107 (2020).

69. Anderson, E. M. et al. Seasonal human coronavirus antibodies are boosted upon SARS-CoV-2 infection but not associated with protection. Preprint at medRxiv https://doi.org/10.1101/2020.11.06.20227215 (2020).

70. Tso, F. Y. et al. High prevalence of pre-existing serological cross-reactivity against SARS-CoV-2 in sub-Sahara Africa. Int. J. Infect. Dis. 102, 577-583 (2020).

71. Rostad, C. A. et al. Quantitative SARS-CoV-2 serology in children with multisystem inflammatory syndrome (MIS-C). Pediatrics 146 , e2020018242 (2020).

72. Weisberg, S. P. et al. Distinct antibody responses to SARS-CoV-2 in children and adults across the COVID-19 clinical spectrum. Nat. Immunol. https://doi. org/10.1038/s41590-020-00826-9 (2020).

73. Pierce, C. A. et al. Immune responses to SARS-CoV-2 infection in hospitalized pediatric and adult patients. Sci. Transl. Med. 564, eabd5487 (2020).

74. Berghöfer, B. et al. TLR7 ligands induce higher IFN- $\alpha$ production in females. J. Immunol. 177, 2088-2096 (2006).

75. Klein, S. L., Marriott, I. \& Fish, E. N. Sex-based differences in immune function and responses to vaccination. Trans. R. Soc. Trop. Med. H. 109, 9-15 (2015).

76. Klein, S. L., Jedlicka, A. \& Pekosz, A. The Xs and Y of immune responses to viral vaccines. Lancet Infect. Dis. 10, 338-349 (2010).

77. Webb, K. et al. Sex and pubertal differences in the type 1 interferon pathway associate with both $\mathrm{X}$ chromosome number and serum sex hormone concentration. Front Immunol. 9, 3167 (2019).

78. Kollmann, T. R., Levy, O., Montgomery, R. R. \& Goriely, S. Innate immune function by Toll-like receptors: distinct responses in newborns and the elderly. Immunity 37, 771-783 (2012).

79. Mertz, D. et al. Pregnancy as a risk factor for severe outcomes from influenza virus infection: a systematic review and meta-analysis of observational studies. Vaccine 35, 521-528 (2017).

80. Pido-Lopez, J., Imami, N. \& Aspinall, R. Both age and gender affect thymic output: more recent thymic migrants in females than males as they age. Clin. Exp. Immunol. 125, 409-413 (2001).
81. Saso, A. \& Kampmann, B. Vaccine responses in newborns. Semin. Immunopathol. 39, 627-642 (2017)

82. Lagunas-Rangel, F. A. Neutrophil-to-lymphocyte ratio and lymphocyte-to-C-reactive protein ratio in patients with severe coronavirus disease 2019 (COVID-19): a meta-analysis. J. Med. Virol. 92 , 1733-1734 (2020).

83. Li, J. et al. Neutrophil-to-lymphocyte ratio positively correlates to age in healthy population. J. Clin. Lab Anal. 29, 437-43 (2014).

84. Yilmaz, H. et al. Usefulness of the neutrophil-to-lymphocyte ratio to prediction of type 2 diabetes mellitus in morbid obesity. Diabetes Metab. Syndr. Clin. Res. Rev. 9, 299-304 (2015).

85. Molony, R. D. et al. Aging impairs both primary and secondary RIG-I signaling for interferon induction in human monocytes. Sci. Signal. 10, eaan2392 (2017)

86. McGonagle, D., Sharif, K., O’Regan, A. \& Bridgewood, C. The role of cytokines including interleukin-6 in COVID-19 induced pneumonia and macrophage activation syndrome-like disease. Autoimmun. Rev. 19, 102537 (2020)

87. Kang, R. et al. HMGB1 in health and disease. Mol. Asp. Med. 40, 1-116 (2014).

88. Gao, Y., Chen, Y., Liu, M., Shi, S. \& Tian, J. Impacts of immunosuppression and immunodeficiency on COVID-19: a systematic review and meta-analysis. J. Infection. 81, 93-95 (2020).

89. Fung, M. \& Babik, J. M. COVID-19 in immunocompromised hosts: what we know so far. Clin. Infect. Dis. ciaa863 (2020).

90. Robilotti, E. V. et al. Determinants of COVID-19 disease severity in patients with cancer. Nat. Med. 26, 1218-1223 (2020).

91. Quinti, I. et al. A possible role for B cells in COVID-19?: lesson from patients with Agammaglobulinemia. J. Allergy Clin. Immunol. 146, 211-213 (2020).

\section{Acknowledgements}

I thank clinical colleagues, lab members and collaborators for fruitful discussions. I also acknowledge the scientific community for openly engaging in discussions on social media, for sharing ideas and collaborating to mitigate the damage of this ongoing crisis.

\section{Competing interests}

The author is a cofounder of Cytodelics AB, Stockholm, Sweden, and is scientific advisor for Scailyte AG, Zurich, Swizerland, and Kancera AB, Stockholm, Sweden.

\section{Additional information}

Correspondence should be addressed to P.B.

Peer review information Hannah Stower was the primary editor on this article and managed its editorial process and peer review in collaboration with the rest of the editorial team.

Reprints and permissions information is available at www.nature.com/reprints. Publisher's note Springer Nature remains neutral with regard to jurisdictional claims in published maps and institutional affiliations.

(C) Springer Nature America, Inc. 2021 